\title{
The Generalization of Orthogonal Frequency Division Multiplexing With Subcarrier Power Modulation to Quadrature Signal Constellations
}

\author{
JEHAD M. HAMAMREH ${ }^{1}$, and ABDULWAHAB HAJAR ${ }^{1}$ \\ ${ }^{1}$ J. M. Hamamreh, and A. Hajar are with WISLAB-TELENG for Wireless Research at the Department of Electrical-Electronics Engineering, Antalya Bilim \\ University, Antalya, Turkey. (web: https://sites.google.com/view/wislab // email: jehad.hamamreh@antalya.edu.tr) \\ Corresponding author: Jehad M. Hamamreh (e-mail: jehad.hamamreh@gmail.com).
}

The Matlab simulation codes used to generate the results in this paper can be found at https://researcherstore.com/. Part of this research has been presented at the 2019 ASYU conference [1]. This research was partly funded by TUBITAK under Grant/Award Number: 119E408.

\begin{abstract}
A novel modulation technique termed as orthogonal frequency division multiplexing with subcarrier power modulation (OFDM-SPM) has been proposed for achieving spectral-efficient data transmission in wireless communication systems. OFDM-SPM utilizes the power of each subcarrier in an OFDM block as an extra degree of freedom to convey extra information bits besides the bits transmitted by conventional signal modulation. OFDM-SPM has originally been introduced with binary phase shift keying (BPSK) symbol modulation, and was shown to provide great gains and various merits such as doubling the spectral efficiency, reducing transmission power and transmission times by half. Displaying its capabilities as a scheme to be adopted for future wireless communication systems, a question detrimental to the adoption of OFDM-SPM has yet to be answered. This is whether the gains that OFDM-SPM brings persist when paired with higher order modulation schemes, especially two dimensional signal constellation schemes such as M-ary PSK. In this paper, OFDM-SPM is paired with quadrature phase shift keying (QPSK) symbol modulation as an example of a higher order two dimensional modulation scheme. The performance analysis of this scheme along with its numerical simulations are carried out where the bit error rate (BER) and throughput performances of the scheme are given in both an additive white Gaussian noise (AWGN), and multipath Rayleigh fading channels. These simulations are done for different power allocation policies. Unlike other 3D modulation methods, the results show that OFDM-SPM can be used with higher order modulation schemes while maintaining all the gains exhibited in OFDM-SPM with BPSK. This gives OFDM-SPM a unique advantage when compared to other 3D modulation schemes such as OFDM-IM and OFDM-SNM, which lose the gain in spectral efficiency as the modulation order becomes higher. Furthermore, the results of OFDM-SPM with QPSK were compared to that of conventional OFDM with 16-QAM symbol modulation. OFDM-SPM displayed superiority both in terms of BER and throughput achieving a gain of approximately $2.5-3 \mathrm{~dB}$. These findings clearly point out that OFDM-SPM is a promising modulation scheme, which should be investigated more vigorously and considered as a strong candidate for adoption in future $6 \mathrm{G}$ and beyond wireless communication systems.
\end{abstract}

INDEX TERMS BER, IoT, Multipath Rayleigh Channel, OFDM, Power Modulation, Spectral Efficiency, Throughput, Wireless Communication, Beyond 5G, 6G, Data Rates, BER, Delay, Power Saving.

\section{INTRODUCTION}

$\mathbf{T}$ HE next generation of communication systems, namely $5 \mathrm{G}$, is soon to be realized, and the need for $6 \mathrm{G}$ technology furthermore, seems inevitable. Particularly, unlike the gen- erations preceding it, $5 \mathrm{G}$ specifications have been catered to various different use cases and applications as required by the current technological era we live in. As such 5G systems have introduced novelties in many technological fields. One of the 
most significant characteristics of $5 \mathrm{G}$, is the great data rates it is able to achieve. Many upcoming data hungry applications requiring data rates unable to be matched by $4 \mathrm{G}$ and $4.5 \mathrm{G}$ systems have emphasized the need of systems capable of providing much higher data rates. On the other hand, the upcoming $6 \mathrm{G}$ generation of communication systems, brings use cases with simultaneous requirements that $5 \mathrm{G}$ technology is unable to meet. Where it is expected to serve technologies such as telemedicine, mixed reality, augmented reality, haptics, brain-machine interfaces and others as [2] and [3] clearly show. These technologies require high spectral efficiency and data rates, low complexity and good reliability simultaneously, thus posing a set of requirements which $5 \mathrm{G}$ technology is unable to handle and emphasizing the need for new technologies capable of serving these applications.

Working towards improving spectral efficiency and data rates, various approaches have been considered. A rather conventional approach is by means of cell densification. This would enable the enhancement of the data rate within a specific area, namely the area within the reach of the aforementioned cells, additionally, this is a useful approach to follow in scenarios of high traffic demand. Cell densification however results in high costs of site rentals and installations, furthermore, laying more cells in a network is a time consuming process, and its given effect is bounded to a specified area. Another technology that has been given increasing importance recently is Millimeter-wave transmission. mmwave transmission allows for higher data rates, by using portions of the frequency spectrum that were previously unused. This leads to great improvements in the data rate, however the propagation characteristics of these waves has hindered its immediate deployment. Where mm-waves can only propagate through short distances and are extremely sensitive to external variables. Another approach has been considering multiplexing techniques other than Orthogonal Frequency Division Multiplexing (OFDM), these methods improve the spectral efficiency in the area they are utilized collectively rather than improving the spectral efficiency exhibited per user. Massive multiple-input-multiple-output (MIMO) and Non-Orthogonal Multiple Access (NOMA) have both been proposed as novel multiplexing techniques. While found to be promising, these techniques result in some complications such as more complex digital signal processing in the transceiver, large latency and low energy utilization efficiency [4]. A scheme which has shown notable potential in providing gains in terms of spectral efficiency and thus data rate is in-band full-duplex, however this scheme has been obstructed by the self-interference it introduces [5].

Given that OFDM has long been used in the technology of today, and was essentially the backbone of $3 \mathrm{G}$ and $4 \mathrm{G}$ communication systems, another approach which has been taken to improve data rates and spectral efficiency is pairing the conventional OFDM waveform with an auxiliary modulation technique capable of laying ground for an extra dimension in which data can be interpreted thus transmitting more data per OFDM symbol. In the literature, schemes such as subcarrier- index modulation OFDM (SIM-OFDM), spatial modulation OFDM (SM-OFDM), OFDM with subcarrier number modulation (OFDM-SNM) and OFDM with index modulation (OFDM-IM) have been reported [6]. A comprehensive study comparing these modulation techniques in terms of their working principles, and their performance with respect to defined metrics has been done in [6].

These techniques bring alongside the conventional amplitude and phase of a symbol, a third characteristic which can be manipulated to introduce an extra data carrying dimension. For instance, the indices of transmitting antennas and active subcarriers of an OFDM block have been used in SM-OFDM [7] and SIM-OFDM [8] respectively as an extra degree of freedom which can be used for the encoding and decoding of extra bits to be transmitted. OFDM-IM [9], similarly uses the indices of the active subcarriers of smaller sub-blocks to convey extra data bits. OFDM-SNM [10] conveys data by manipulating the number of active subcarriers within smaller OFDM sub-blocks. While these techniques were able to achieve some gain in terms of spectral efficiency, each has its own shortcomings some of which are inherent due to the working principles of the scheme itself. Where some of these schemes require a highly complex transceiver structure, and others, such as schemes which depend on the indices of the active subcarriers have shown an inability to provide the same gains at high transmission rates, as such the gain of schemes such as IM-OFDM and SIM-OFDM has been reported to erode when paired with higher order symbol modulation techniques [11], this claim has been confirmed by [12] as well. This results in ultimately limiting the scope of applicability of these schemes, and bounds them to select applications and use cases. Furthermore, the working principles of some schemes such as OFDM-SNM and OFDMIM dictate the inactivation of a portion of the subcarriers of the OFDM blocks or sub-blocks. This conceptually leads to a decrease in the data rate. However, this is compensated for by the data transmitted by the additional dimension which was established, or by other auxiliary techniques introduced by follow-up research. For example, aiming to enhance OFDMIM, researchers were able to devise schemes which utilize the subcarrier indices of the OFDM block, without resorting to the need of deactivating some of the subcarriers. Multiple Mode (MM)-OFDM-IM [13] and Dual Mode (DM)-OFDM with Index modulation [14], are examples of this which evidently achieve better spectral efficiency than OFDM-IM. Further research has also led to the extension of some of these concepts to a wide variety of different domains. This has lead to the emergence of a multitude of schemes within each domain. As such, many surveys have been done on the concept of index modulation in OFDM, reviewing the various domains to which it has been applied. In [15], it is shown that the concept of index modulation has been to extended to the space [7], frequency [9], space-frequency [16] and space-time [17] domains. Furthermore, [18] has shown that the concept of index modulation has also been extended to the angle domain, where Polarization Shift Keying (PolarSK) 
[19] and other schemes were proposed. This scheme uses the polarization states to convey index information in the angle domain, thus achieving a high spectral gain at a minor cost in terms of hardware complexity [18]. Other schemes which utilized index modulation in the angle domain followed, bringing higher spectral efficiency and better BER performance [18]. Index modulation has also been extended to the channel domain [20], where Channel Domain Index Modulation (CD-IM) also known as Media Based Modulation (MBM) [21] has been proposed. Contrary to other index modulation techniques, MBM focuses the IM operations on the variable channel states, rather than the antennas, subcarriers or time slots at the TX/RX in order to convey additional information bits. However, while MBM is able to achieve great gains in terms of spectral and energy efficiency, it faces several challenges, such as the large size of the training overhead required for acquiring the channel states, which are essential to the working mechanisms of MBM [20]. Finally, further research has also improved the performance of OFDM-SNM, where Enhanced OFDM-SNM scheme has been proposed [22] to improve the reliability performance of the original OFDM-SNM scheme.

Furthermore, Orthogonal Frequency Division Multiplexing with Subcarrier Power Modulation (OFDM-SPM) has recently been proposed ${ }^{1}$ in [24], [25]. This scheme also adds a third dimension to carry extra data bits which is the power of the OFDM subcarriers. In [24], [25], OFDM-SPM was introduced with BPSK symbol modulation. It displayed various merits in terms of spectral efficiency, energy efficiency, transmission times, complexity and is characterized by the absence of any form of inherent error propagation. However, a critical question that appears at the forefront of what is to be investigated regarding OFDM-SPM is whether these performance gains persist when OFDM-SPM is paired with higher order symbol modulation techniques such as M-ary PSK. This is critical to know because other schemes such as OFDM-IM and OFDM-SNM have shown a decay in spectral gain when paired with higher order modulation schemes. As such, this paper pairs OFDM-SPM with QPSK symbol modulation as an example of a higher order two-dimensional modulation scheme and investigates its ensuing studies regarding validity and performance. The main contributions of this paper can be summarized as:

- Pairing OFDM-SPM with a two dimensional modulation scheme (QPSK), explaining how SPM can be applied to a two dimensional symbol modulation scheme, and investigating whether pairing OFDM-SPM with higher order modulation schemes leads to a decay in the scheme's gain.

- The performance analysis of OFDM-SPM with QPSK in terms of power efficiency, spectral efficiency and other metrics is provided.

\footnotetext{
${ }^{1}$ It has been reported that power has been used as an extra degree of freedom along with index modulation in the domain of optical communications, yielding however only marginal gains in the spectral efficiency as in [23].
}

- Providing comprehensive numerical simulation results for OFDM-SPM in terms of BER and throughput under different use cases and for both the power saving and power reallocation policies.

- A comparison between conventional OFDM with 16QAM symbol modulation and OFDM-SPM with QPSK in terms of BER and throughput is carried out with numerical simulations. This comparison is done under fair conditions where an OFDM-SPM block uses the same power as that of an OFDM with 16-QAM block.

OFDM-SPM with QPSK has displayed superiority to conventional OFDM with QPSK in terms of various metrics. Overall, OFDM-SPM brings the following advantages when compared to conventional OFDM with QPSK:

- OFDM-SPM with QPSK attains a doubling in the spectral efficiency and thus data rate of the system, when compared to that of conventional OFDM with QPSK. As such, OFDM-SPM requires only half the number of subcarriers required by conventional OFDM to transmit the same number of bits, equally stated it can be said that OFDM-SPM with QPSK offers a data rate equal to that of an OFDM system employing 16-QAM symbol modulation.

- OFDM-SPM with QPSK uses half the transmission power used by conventional OFDM. Additionally, it also reduces the overall transmission delay of the system. This is due to the fact that OFDM-SPM with QPSK uses half the number of subcarriers used by conventional OFDM, which translates to fewer resources in the time domain as well. This reduction of the number of subcarriers used also gives OFDM-SPM the potential to reduce the IFFT size to half of its original size, thus reducing the overall complexity of the system.

- As OFDM-SPM uses fewer subcarriers thus saving power, it is characterised with flexibility in the sense that this saved power can be reallocated to improve the error performance of the system, or saved according to the needs of the application.

Additionally, since OFDM-SPM with QPSK achieves a data rate similar to that of conventional OFDM with 16-QAM symbol modulation, this encourages a comparison between the two, OFDM-SPM displayed the following merits:

- Under fair conditions, where an OFDM-SPM symbol with QPSK is given the same power as that of OFDM with 16-QAM, OFDM-SPM with QPSK is shown to display a $2.5-3 \mathrm{~dB}$ gain in the BER of the system while also achieving 3-4dB gain over the throughput of a 16QAM transmission system.

- A comparison between OFDM-SPM with QPSK and conventional OFDM with 16-QAM, where OFDMSPM uses half the power used by conventional OFDM is given as well. It was found that OFDM-SPM with QPSK provides a near identical performance to that of conventional OFDM with 16-QAM while requiring only the power used by conventional OFDM with 
QPSK which is specifically a half of the power used by conventional OFDM with 16-QAM, thus reducing the power consumption greatly. This also characterizes OFDM-SPM with flexibility once again, as it gives the user the option between using OFDM-SPM with QPSK rather than conventional OFDM with 16-QAM to attain the same performance while reducing the transmission power significantly, or using OFDM-SPM with QPSK with the same power as that of conventional OFDM with 16-QAM to provide a BER and throughput which is superior to that of conventional OFDM with 16-QAM.

Overall OFDM-SPM can be described as a highly spectrally efficient system, which adds little complexity to the transceiver structure of the system, and is actually capable of reducing it. Furthermore, OFDM-SPM seems to be an ideal fit for applications in the IoT domain due to its low complexity, low power requirements, small time delays, flexibility and spectral efficiency, meeting many of the various demands required by IoT applications. This and the above-mentioned gains which can be achieved by OFDM-SPM display how it can be utilized to serve $5 \mathrm{G}$ applications. Furthermore, the advantageous characteristics of OFDM-SPM, and the findings from the comparisons emphasize it as being a potential candidate for $6 \mathrm{G}$ applications such as mixed reality, real time gaming, high definition video streaming, and brainmachine interfaces, all of which require low latency, good reliability, low complexity and extremely high throughput, which OFDM-SPM in one way or another works to meet.

The remaining sections of this paper are organized as follows. The OFDM-SPM system model and how it is conceived with a two dimensional constellation scheme (QPSK) is explained and illustrated in Section II. Performance analysis is carried out in section III. Performance demonstration is given in section IV. Finally, the conclusion and future promising research works are given in Section V.

\section{OFDM-SPM: SYSTEM MODEL}

In this section, we explain and illustrate in detail the transmitter and receiver designs of the proposed OFDM-SPM scheme.

\section{A. THE TRANSMITTER DESIGN}

The main concept of OFDM-SPM is to manipulate the power of each subcarrier in the OFDM block to transmit more than one bit per subcarrier. The general architecture of the transmitter is depicted in Fig. 1.

Unlike conventional OFDM, OFDM-SPM splits the serial input bit stream of length $2 n \log _{2} M$ into two sub-streams of $n \log _{2} M$ bits, where $n$ is the number of subcarriers in the OFDM block used to carry data and $M$ represents the modulation order, which in the case of QPSK is 4. As such, $\log _{2} M$ is the number of bits per symbol according to the symbol modulation scheme used.

As can be seen from Fig 1, one of the substreams determines the power of the in-phase and quadrature subcarriers of the OFDM block, where the $(2 i-1)^{t h}$ bit determines the

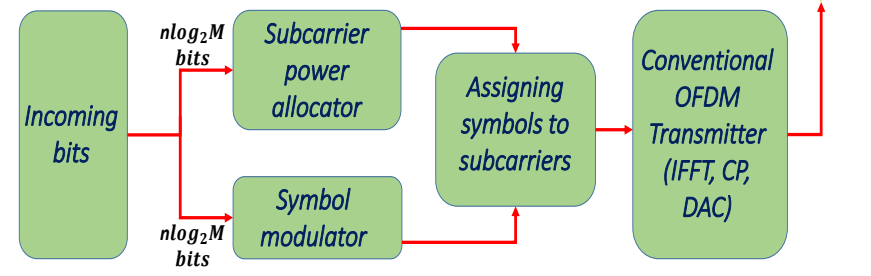

FIGURE 1. Transmitter structure of OFDM-SPM

power level of the $i^{\text {th }}$ in-phase subcarrier, and the $2 i^{\text {th }}$ bit determines the power of the $i^{\text {th }}$ quadrature subcarrier utilized to carry data where $i$ varies from 1 to $n$. Consequently, a '00' corresponds to setting the power of both the in-phasae and quadrature subcarriers to low power, a '11' corresponds to setting both to high power, a '01' corresponds to setting the in-phase subcarrier to high power and the quadrature subcarrier to low power , and a '10' indicates the opposite. The second sub-stream of bits is modulated using regular QPSK modulation. The QPSK symbols are then assigned to their respective subcarriers. Finally, the symbols go through the remaining steps of the conventional OFDM transmission process, including inverse fast Fourier transform (IFFT), cyclic prefix (CP) addition, and digital-to-analog conversion (DAC).

Thus, OFDM-SPM results in 4 different constellation points for each of the in-phase and quadrature subcarriers as in Fig. 2. Since the in-phase and quadrature subcarriers are orthogonal to each other, this gives us a total of 16 constellation points for this scheme.

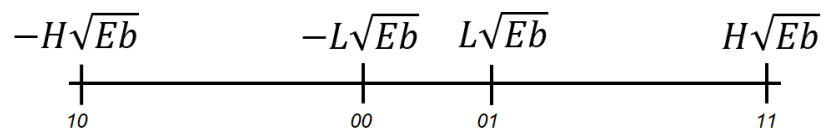

FIGURE 2. Constellation points of in-phase / quadrature subcarriers of OFDM-SPM with QPSK

As QPSK can equally be seen as two orthogonal branches of BPSK, In Fig. 2, '00' can be seen as corresponding to a low power in-phase/quadrature subcarrier carrying a ' 0 ' modulated by BPSK (the corresponding in-phase/quadrature component of the QPSK symbol), '11' corresponds to a high power subcarrier carrying a ' 1 ' modulated by BPSK, '01' corresponds to a low power subcarrier carrying a '1' modulated by BPSK, and vice versa. Keeping in mind that we have two orthogonal subcarriers namely the in-phase and quadrature, we see that OFDM-SPM with QPSK by controlling the power levels of the in-phase and quadrature 
subcarriers of the OFDM block gives us a total of 4 bits per symbol, where each component in-phase or quadrature carries 2 bits. Furthermore, in Fig. $2 E_{b}$ denotes the energy per bit, and $L$ and $H$ denote the amplitude levels corresponding to the low and high power levels of the subcarriers, respectively.

Aiming to optimize the performance of the scheme in terms of BER and throughput, the $H$ and $L$ values were determined. This was done while taking into consideration that the found values must not lead the average energy per OFDM block to be more than that of conventional OFDM with QPSK. This constraint makes sure that OFDM-SPM not only brings a great gain in spectral efficiency, but also requires no extra power, and was found to rather save power when compared to conventional OFDM, this will be displayed by the following example.

Example: A simple scenario to fully illustrate the merits of OFDM-SPM, and give a clear comparison between it and conventional OFDM is given. Assuming we were to transfer 208 bits, let us simply investigate the resources required by each scheme. If we were to use conventional OFDM with QPSK, 104 subcarriers would be required, each requiring power $P$ and bandwidth $W$, resulting totally in $104 P$ and $104 \mathrm{~W}$ power and bandwidth respectively. However, by utilizing OFDM-SPM, 52 subcarriers would be able to transfer the same number of bits, resulting in a total power and bandwidth of $52 P$ and $52 \mathrm{~W}$.

As illustrated in Fig. 3, OFDM-SPM manipulates the power of the in-phase and quadrature subcarriers of the OFDM block to convey twice the amount of data bits thus doubling the spectral efficiency where conventional OFDM as per the figure uses $2 W$ bandwidth, whereas OFDMSPM only requires $W$ bandwidth for the same number of bits transmitted. Additionally, OFDM-SPM reduces the total power usage by half as a consequence of using only half the number of subcarriers used by conventional OFDM. This saved power provides OFDM-SPM with flexibility since this power can either be saved or reallocated according to the requirements of the application. Applications requiring low complexity and low power can benefit from this merit of OFDM-SPM, thus presenting OFDM-SPM as a favorable modulation scheme for applications such as IoT. For applications requiring a less erroneous BER, the saved power can be reallocated resulting in an enhanced BER.

The low and high power levels of the in-phase and quadrature subcarriers which optimize the resulting BER of the system are found by means of a successive process of exhaustive trial and error experiments. The power levels in the cases of power saving, and power reallocation policies [8] are defined according to Eq. (1) and (2), respectively, where the equations refer to bits carried by either subcarrier, in-phase or quadrature and apply to both.

$$
\begin{aligned}
& L^{2}+H^{2}=2 E_{b} \\
& L^{2}+H^{2}=4 E_{b}
\end{aligned}
$$
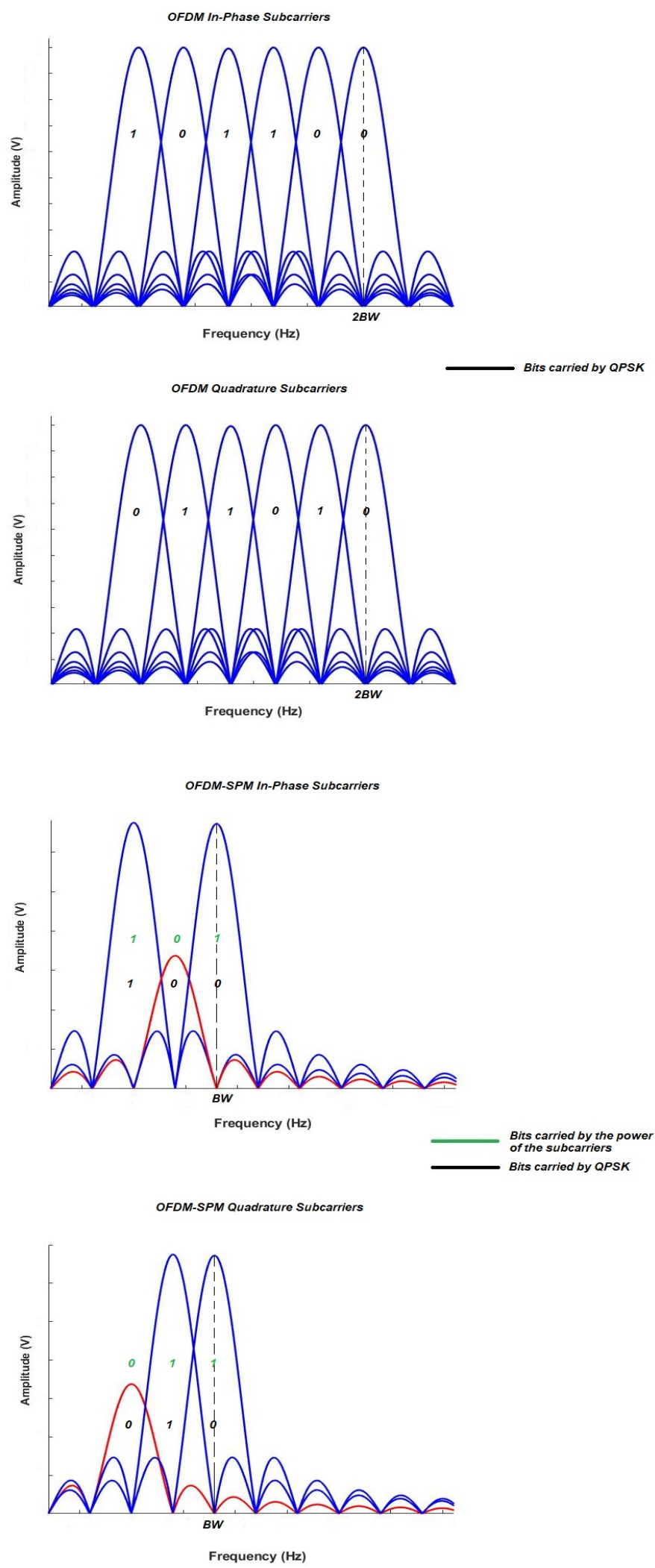

FIGURE 3. Comparing OFDM-SPM and conventional OFDM 
Since OFDM-SPM with QPSK was compared to conventional OFDM with 16-QAM, it was necessary to make the average power per OFDM block of both schemes equal, so as to ensure a fair comparison. As such, the optimal $H$ and $L$ values of OFDM-SPM were found according to Eq. (3)

$$
L^{2}+H^{2}=8 E_{b}
$$

In the case of power saving for example, Eq. (1) is followed to determine the values of $H$ and $L$. By setting the value of $H$ to an arbitrary value, the corresponding value of $L$ can be found as follows:

$$
L=\sqrt{2 E_{b}-H^{2}}
$$

The optimal values of $H$ and $L$ in the case of power reallocation and when OFDM-SPM is compared to OFDM with 16-QAM were found in a similar manner, but using Eq. (2) and Eq. (3) respectively instead of Eq. (1).

Simulations were run for different values of $H$ and $L$, and the values resulting in the optimal BER were obtained as $H=$ 1.35 and $L=0.4213$ in the case of power saving, $H=1.918$ and $L=0.5668$ in the case of power reallocation, and $H=$ 2.7 and $L=0.8426$ in the case of Eq. (3).

\section{B. CHANNEL MODEL}

The channel is assumed to be a slowly varying, Rayleigh multi-path fading channel with $L$ exponentially decaying taps, denoted by $\boldsymbol{h}=\left[h_{0}, h_{1}, \cdots, h_{(L-1)}\right]$ [10], [26]. As such the received symbols are given as:

$$
\boldsymbol{y}=\boldsymbol{x} \circledast \boldsymbol{h}+\boldsymbol{n},
$$

where $\circledast$ represents the convolution operation. Also, the symbols $\boldsymbol{x}, \boldsymbol{y}, \boldsymbol{h}$ and $\boldsymbol{n}$ are vectors representing the transmitted time domain samples, received samples in the time domain, the channel impulse response, and the additive white Gaussian noise, respectively. Furthermore, the $\boldsymbol{n}$ vector can be statistically characterized by

$$
\boldsymbol{n} \sim \mathcal{N}\left(0, N_{0}\right),
$$

where the elements (noise samples) of $\boldsymbol{n}$ has zero mean and variance equal to $N_{0}$. Additionally, the channel is slowly varying in time such that it is assumed to be constant for multiple OFDM symbols duration before it changes independently in the subsequent time intervals.

\section{THE RECEIVER DESIGN}

OFDM-SPM is one of the few schemes capable of adding a third dimension, while adding minor complexity to the receiver structure. This is a significant merit for OFDMSPM (i.e., highly desirable for future wireless standards). The receiver structure is shown in Fig. 4. Initially, the scheme goes through many of the processes of conventional OFDM reception such as ADC, CP removal, FFT, etc. At the symbol detection phase however, the detection is done in two separate demodulation blocks in a parallel manner. The first detects the bits conveyed by the power levels of the in-phase

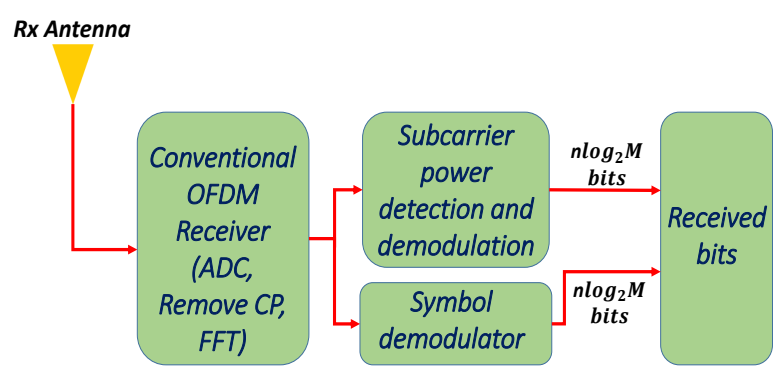

FIGURE 4. Receiver structure of OFDM-SPM

/ quadrature subcarriers, and encompasses non-coherent detection. This is done by means of measuring the power of each subcarrier, and comparing it with a specified threshold $T$. For optimum performance, this threshold is determined as the power corresponding to the midpoint between the high and low-level amplitudes given to the subcarriers. If the power of a subcarrier is lower than the threshold, a low power subcarrier is detected, hence interpreting a ' 0 ' and vice versa. The other demodulator performs conventional QPSK demodulation to the symbols.

The aforementioned midpoint and threshold $T$ used for non-coherent detection are given by Eq. (6) and (7), as follows.

$$
\begin{gathered}
\text { midpoint }=\left(\frac{L+H}{2}\right) \\
T=\left(\frac{L+H}{2}\right)^{2}
\end{gathered}
$$

It is important to note that the detection process of the bits conveyed by the subcarrier power by means of thresholding adds little complexity to the receiver structure of the system. In terms of complexity, this gives OFDM-SPM an advantage over other schemes such as OFDM-SNM, OFDM-IM and SIM-OFDM which have employed either a maximum likelihood (ML) detectors for optimum performance, or a $\log$ likelihood ratio (LLR) detector for reduced complexity [6]. Both of these, however, introduce far more complexity to the receiver structure than that of OFDM-SPM.

\section{PERFORMANCE ANALYSIS}

\section{A. POWER AND SPECTRAL EFFICIENCY}

Unlike other schemes, in OFDM-SPM, the number of subcarriers which are active in each OFDM block does not vary. In particular, all the subcarriers are utilized for data transmission, which is why measuring the spectral and power efficiency of OFDM-SPM is rather simple. Referring to Fig. 3 , it is evident that OFDM-SPM always attains a doubling 
of the spectral efficiency, and a reduction of the transmission power by half. Furthermore, when compared to conventional OFDM with 16-QAM, OFDM-SPM was found to use a half of the power used by conventional OFDM with 16QAM while achieving near identical performance in terms of spectral efficiency, BER and throughput.

\section{B. COMPLEXITY}

One of the main advantages of OFDM-SPM when compared to other schemes such as OFDM-IM, and OFDM-SNM is the minor computational complexity which its transceiver structure adds to the traditional transceiver structure of conventional OFDM. As the order of computational complexity of OFDM-SPM, is in the order of $O(M)$, where $\mathrm{M}$ is the modulation order, which is similar to that of conventional OFDM. As shown in [6], OFDM-SNM exhibits a higher complexity than OFDM-SPM. OFDM-IM furthermore, displays similar complexity, where it is also in the order of $O(M)$, when implementing an LLR detector. However, when considering the number of FLOPs (floating point operations) which both OFDM-IM and OFDM-SPM add to the conventional OFDM transmission and reception processes, it can be shown that OFDM-IM with its LLR detector adds far more complex operations, this is because the calculation of the LLR value for each detected bit requires the a-posteriori probabilities of all the possible transmitted signal vectors [27], whereas OFDM-SPM simply requires the comparison of the power of the subcarriers of each symbol with a given threshold which adds a negligible number of FLOPs relative to OFDM-IM.

\section{BIT ERROR RATE (BER)}

Since QPSK can be seen as two identical orthogonal BPSK branches, the average BER of OFDM-SPM with QPSK will be identical to that of OFDM-SPM with BPSK [24], as the average of two identical quantities is the respective quantity itself. Similar to OFDM-SPM with BPSK there are two possible causes of errors. Firstly, an error arising from the detection of the bits of the modulated symbols carried by the inphase/quadrature subcarriers. The second cause of a possible error is the detected power level of each in-phase/quadrature subcarrier, where the noise and fading of the channel might attenuate or amplify the power of a subcarrier, causing a high power subcarrier to be detected as a low power subcarrier and vice versa.

Fig. 2 clearly shows that the power of the subcarrier of a QPSK symbol affects how prone to error it is. As such, a QPSK symbol assigned to a low power subcarrier is more likely to be detected in error, as the Euclidean distance between its constellation points is small. Although assigning QPSK symbols to low power subcarriers negatively affects the BER performance of the system, this is compensated for by the error performance of the symbols assigned to high power subcarriers. Since two different cases are posed, the theoretical expression of the BER performance due to QPSK symbol demodulation is then, the average of the BER expressions of the high and low power QPSK schemes. Given that the difference between conventional QPSK and that of OFDM-SPM is the power of the subcarriers. The expressions can be found by simply adding a multiplication factor which simulates this change to the expression of conventional QPSK in both AWGN and Rayleigh fading channels. These expressions are given as:

\section{1) AWGN Channel}

The BER expressions due to the QPSK detection in an AWGN channel are described as follows:

$$
\begin{gathered}
B E R_{H}=\frac{1}{2} \operatorname{erfc}\left(H \sqrt{\frac{E_{b}}{N_{0}}}\right) \\
B E R_{L}=\frac{1}{2} \operatorname{erfc}\left(L \sqrt{\frac{E_{b}}{N_{0}}}\right) \\
B E R_{Q P S K}=\frac{B E R_{L}+B E R_{H}}{2}
\end{gathered}
$$

Additionally, the analytical expression of the BER resulting from the detection of the powers of the subcarriers is mathematically derived and given as:

$$
B E R_{P}=A+B-C
$$

where $A, B$ and $C$ are the final terms in the BER expression after the mathematical derivation and the collection of terms. $A, B$ and $C$ mathematical expressions are found respectively as follows:

$$
\begin{aligned}
A & =\frac{1}{2} \operatorname{erfc}\left(\frac{H-L}{2} \sqrt{\frac{E_{b}}{N_{0}}}\right) \\
B & =\frac{1}{4} \operatorname{erfc}\left(\frac{3 L+H}{2} \sqrt{\frac{E_{b}}{N_{0}}}\right) \\
C & =\frac{1}{4} \operatorname{erfc}\left(\frac{3 L-H}{2} \sqrt{\frac{E_{b}}{N_{0}}}\right) \\
B E R_{O F D M}-S P M & =\frac{B E R_{P}+B E R_{Q P S K}}{2}
\end{aligned}
$$

This theoretical expression displayed by Eq. (15), is plotted against the simulated errors and is found to coincide perfectly with them.

\section{2) Rayleigh Fading Channel}

The BER expressions due to the detection of QPSK in a Rayleigh fading channel for the case of high power and lower symbols are described by the following equations:

$$
\begin{aligned}
& B E R_{H}=\frac{1}{2}\left(1-\sqrt{\frac{H^{2} \frac{E_{b}}{N_{0}}}{1+H^{2} \frac{E_{b}}{N_{0}}}}\right) \\
& B E R_{L}=\frac{1}{2}\left(1-\sqrt{\frac{L^{2} \frac{E_{b}}{N_{0}}}{1+L^{2} \frac{E_{b}}{N_{0}}}}\right) \\
& B E R_{Q P S K}=\frac{B E R_{L}+B E R_{H}}{2}
\end{aligned}
$$


Furthermore, the BER arising from the detection of the power of the in-phase/quadrature subcarriers are given by the following equations:

$$
B E R_{P}=A+B-C,
$$

where $A, B$ and $C$ are the final terms in the BER expression after the mathematical derivation and the collection of terms. $A, B$ and $C$ mathematical expressions are found respectively as follows:

$$
\begin{aligned}
A & =\frac{1}{2}\left(1-\sqrt{\frac{\left(\frac{H-L}{2}\right)^{2} \frac{E_{b}}{N_{0}}}{1+\left(\frac{H-L}{2}\right)^{2} \frac{E_{b}}{N_{0}}}}\right) \\
B & =\frac{1}{4}\left(1-\sqrt{\frac{\left(\frac{H+3 L}{2}\right)^{2} \frac{E_{b}}{N_{0}}}{1+\left(\frac{H+3 L}{2}\right)^{2} \frac{E_{b}}{N_{0}}}}\right) \\
C & =\frac{1}{4}\left(1-\sqrt{\frac{\left(\frac{3 H+L}{2}\right)^{2} \frac{E_{b}}{N_{0}}}{1+\left(\frac{3 H+L}{2}\right)^{2} \frac{E_{b}}{N_{0}}}}\right)
\end{aligned}
$$

Thus, the total average BER of OFDM-SPM with QPSK theoretically is the average of Eq. (18) and (19).

$$
B E R_{O F D M-S P M}=\frac{B E R_{P}+B E R_{Q P S K}}{2}
$$

The expression given by Eq. (23) was plotted and found to fairly coincide with the simulated results.

Due to the similarity of the manner followed in the derivation of the total BER expressions of OFDM-SPM with QPSK in an AWGN and Rayleigh fading channel, it is found sufficient that the detailed derivation of the BER expression of OFDMSPM in a Rayleigh fading channel is given in the Appendix.

It is important to note that, when OFDM-SPM utilizes the power of the in-phase and quadrature subcarriers as an additional data carrying dimension, it does not lead to any kind of error propagation. Error propagation has been exhibited in a number of schemes such as SIM-OFDM [28], where the error propagation stood out as a critical drawback. Furthermore, the detection processes of the QPSK bits and power bits in OFDM-SPM exhibit an independence in the sense that an error in the detection of one does not necessarily lead to an error in the other. This is because the QPSK detection involves detecting the phase of the carried QPSK symbol, whereas the detection of the power bits involves measuring the power of the in-phase and quadrature subcarriers.

\section{PERFORMANCE DEMONSTRATION}

Numerical simulations displaying the BER and throughput performance of OFDM-SPM with QPSK were conducted. Table I shows the simulation parameters used in this study. The system was simulated in both an AWGN and a multipath Rayleigh fading environment. The Rayleigh fading channel is slowly time-varying such that it is assumed to be constant

\begin{tabular}{|c|c|}
\hline Modulation type & BPSK $(M=2)$ \\
\hline IFFT / FFT size & 64 \\
\hline Subcarriers for data $n$ & 52 \\
\hline Symbols allocated for cyclic prefix & 16 \\
\hline Number of inactive subcarriers for out of band emission & 12 \\
\hline Number of OFDM symbols & $2 \times 10^{4}$ \\
\hline Multipath channel delay samples locations & {$\left[\begin{array}{llllllllll}0 & 3 & 5 & 6 & 8\end{array}\right]$} \\
\hline Multipath channel tap power profile (dBm) & {$\left[\begin{array}{lllll}0 & -8 & -17 & -21 & -25\end{array}\right]$} \\
\hline
\end{tabular}
for a block of OFDM symbols, but changes independently from one block to another. The results are displayed under different power allocation policies.
TABLE 1. Simulation Parameters

\section{A. POWER SAVING POLICY}

By referring to Fig. 3, we can see that OFDM-SPM uses only half the number of subcarriers that conventional OFDM would require to send the same number of data bits. Thus, half the power used by conventional OFDM is unused (i.e., saved) by OFDM-SPM. In the power saving policy, this power is saved to match requirements of low power applications (e.g., IoT). This inherently results in a better power efficiency when compared to conventional OFDM. The power levels in the simulation of this case are defined as in Eq. (1), and were found as $H=1.35$ and $L=0.4213$.

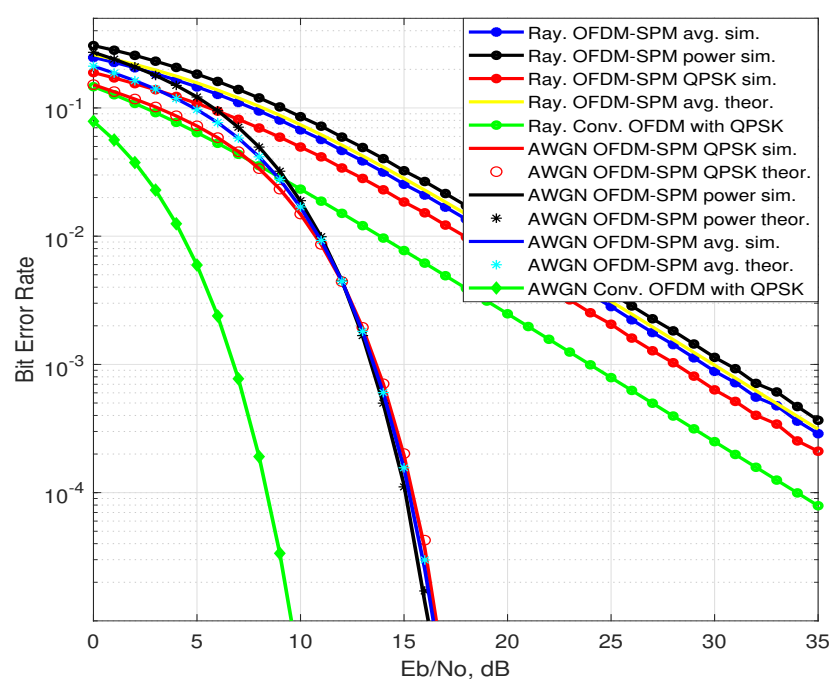

FIGURE 5. BER of OFDM-SPM with power saving

As can be seen from the results in Fig. 5, the overall, average performance of OFDM-SPM when compared to conventional OFDM with QPSK displays a noticeable degradation in the BER both in the case of an AWGN and Rayleigh fading channel; however, compensating for it is an equally significant improvement in the system throughput as can be shown in Fig. 6. It can be seen that the throughput is doubled for high values of SNR amounting to a doubling in the data rate (4bps/subcarrier) in the case of both Rayleigh and AWGN channels. Furthermore, from Fig. 6, OFDM-SPM is able to achieve a throughput of 3.9 (bps/subcarrier) for SNR values as low as 9dB in an AWGN channel, and 16dB in a Rayleigh fading channel. Even at low SNR values, as low as 0dB, OFDM-SPM achieves a data rate of 3.15 (bps/subcarrier) 


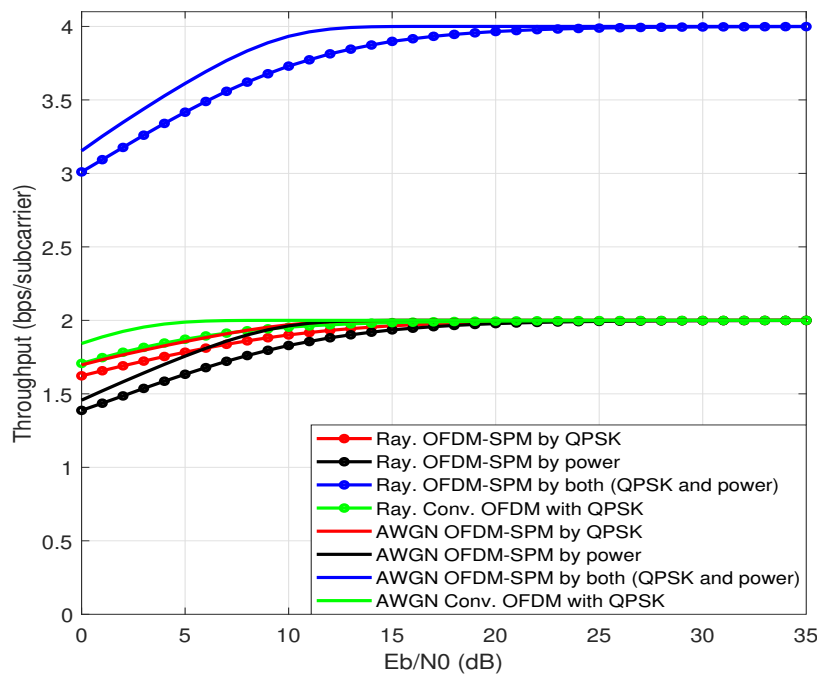

FIGURE 6. Throughput of OFDM-SPM with power saving

and 3 (bps/subcarrier) in an AWGN and Rayleigh fading environment respectively, compared to 1.84 (bps/subcarrier) and 1.71 (bps/subcarrier) of conventional OFDM. More importantly however, this large gain in throughput is achieved while using only half the power used by conventional OFDM, thus characterizing OFDM-SPM as a fit candidate for low power, low complexity applications. It is critical to note that the theoretical expressions derived for OFDM-SPM in both AWGN and fading channels coincide exactly with the simulated findings, thus proving and validating the accuracy of the derived BER.

\section{B. NON-OPTIMIZED POWER REALLOCATION POLICY}

The power which OFDM-SPM saves can be utilized to the scheme's advantage. As the power saving policy shows a degradation in the BER, this saved power can be reallocated to the subcarriers of the OFDM symbol, resulting in an enhanced BER. The method through which the saved power is redistributed amongst the subcarriers will ultimately affect the BER performance. Power reallocation has been investigated for two different cases.

First is the non-optimized case. In this scenario, the gain of OFDM-SPM is clearly displayed. Unlike conventional OFDM, OFDM-SPM can be viewed as a transmission method that provides two streams of data. One of them being the bits carried by the QPSK symbols, and the other being the bits carried by the power of the in-phase and quadrature subcarriers of the OFDM block. In this scheme, power is reallocated such that the bit error rate performance of the bits carried by the QPSK symbols does not degrade but is rather less erroneous than the case of conventional OFDM. This is done by setting the high power level of the subcarriers to $\sqrt{3}$, and the low power level to $L=1$. This QPSK data stream exhibits a bit error rate superior to that of conventional OFDM, this is especially evident in a Rayleigh environment

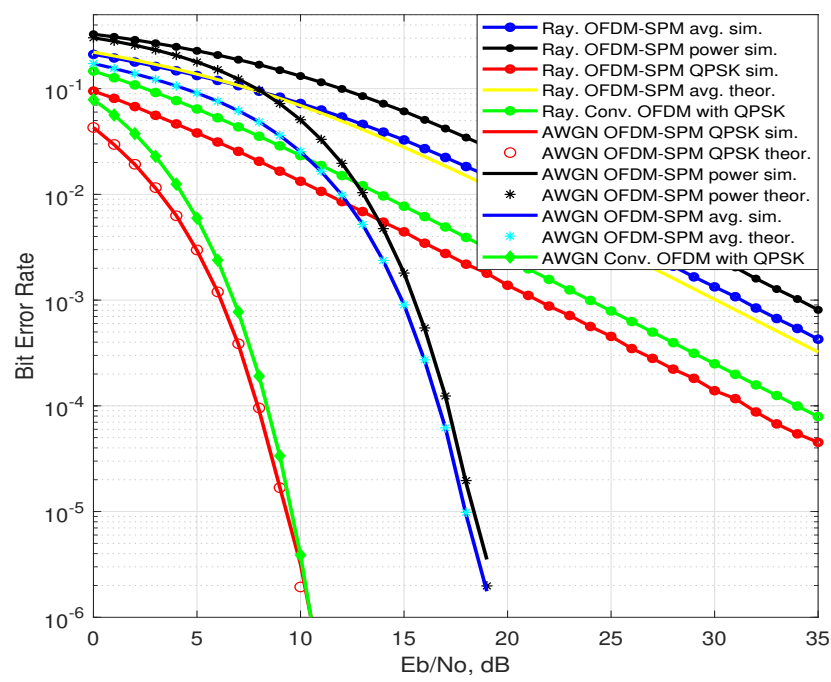

FIGURE 7. BER of OFDM-SPM with non-optimized power reallocation

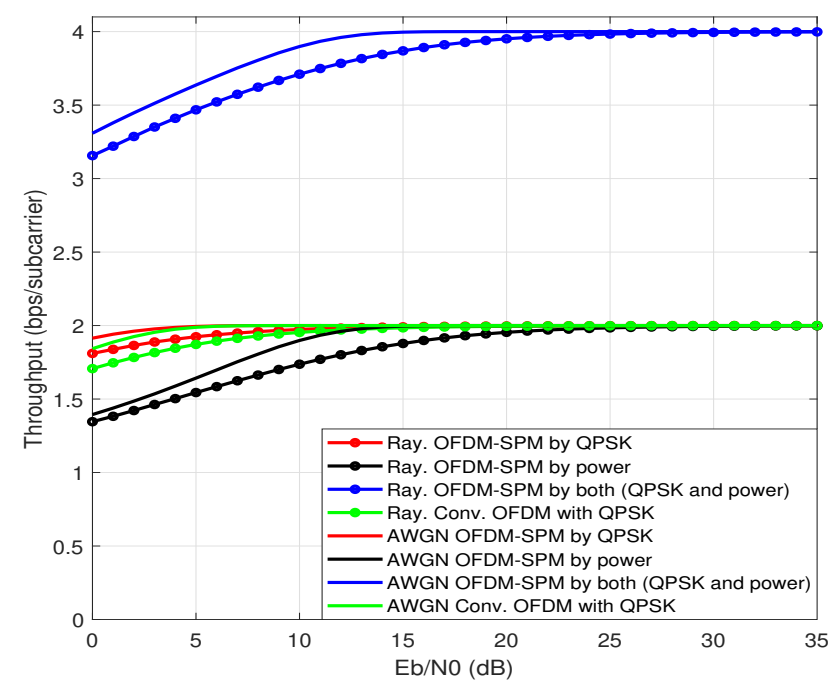

FIGURE 8. Throughput of OFDM-SPM with non-optimized power reallocation

as the gain reaches $2 \mathrm{~dB}$ as can be seen in Fig. 7. This gain is because the high power bits map to constellation points which are further apart than that of conventional OFDM. Furthermore, an additional data stream is provided by the bits carried by the power levels of the subcarriers. Although, this stream exhibits a high frequency of errors, it can be seen as a mere additional benefit to the enhanced QPSK bit error rate. Furthermore, the erroneous bit stream can be assigned to a user application that does not require ultra reliability such as audio streaming services. The theoretical errors of OFDM-SPM in an AWGN channel were found to match the simulated results as well.

Furthermore, the gains in terms of throughput are evident from Fig. 8. Although the power reallocation has not been 
optimized for this case, the throughput achieved still exceeds that of the power saving policy. In this case, for values of SNR as low as $0 \mathrm{~dB}$ OFDM-SPM was able to achieve a throughput of 3.31 (bps/subcarrier), and 3.2 (bps/subcarrier), compared to 1.84 (bps/subcarrier), and 1.71 (bps/subcarrier) of conventional OFDM in an AWGN and Rayleigh fading channel respectively. Furthermore, at high values of SNR a doubling of the throughput is achieved. This basically reflects the scheme's capability of doubling the spectral efficiency of the system.

\section{OPTIMIZED POWER REALLOCATION POLICY}

In this case, the optimal power levels which provide optimal average BER for OFDM-SPM were found according to Eq. (2). Exhaustive trial and error optimization was used to find the corresponding optimal $H$ and $L$ values, which were found as $H=1.918$ and $L=0.5668$. As Fig. 9 shows, the bit error rate performance of the scheme is improved by $3 \mathrm{~dB}$ for a Rayleigh fading channel and approximately $2.5 \mathrm{~dB}$ for an AWGN channel. Although a deterioration in the bit error rate is still observed when compared to conventional OFDM, the overall gains that OFDM-SPM offer can outweigh this degradation. Furthermore, the theoretical BER expressions for OFDM-SPM in an AWGN channel were also found to match with the simulation results for this case.

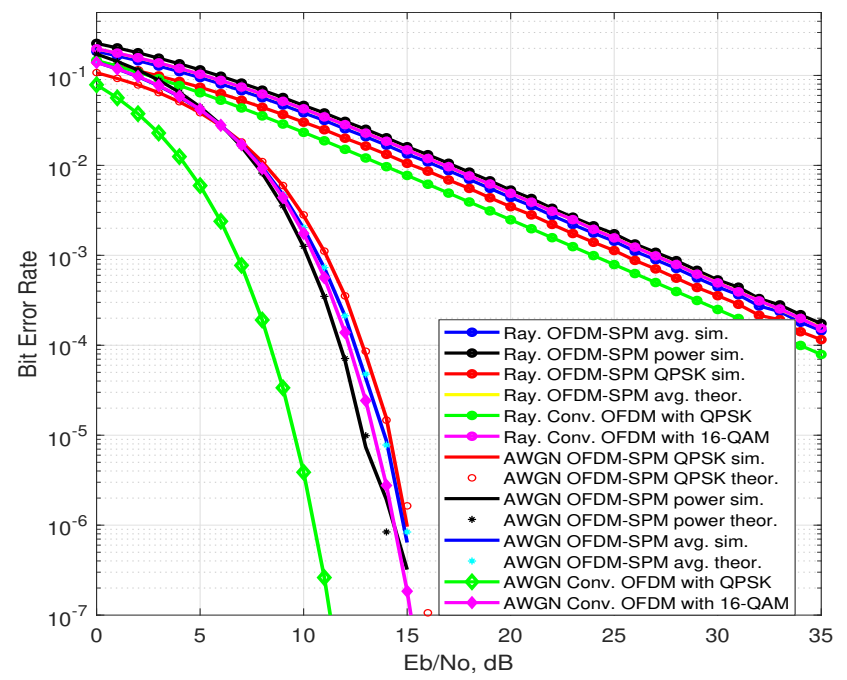

FIGURE 9. BER of OFDM-SPM with optimized power reallocation

Fig. 10 also displays the gain of OFDM-SPM by the throughput performance of OFDM-SPM with optimized power reallocation, where for values as low as $0 \mathrm{~dB}$, throughputs of 3.44 (bps/subcarrier) and 3.26 (bps/subcarrier) are achieved compared to 1.84 (bps/subcarrier) and 1.71 (bps/subcarrier) of conventional OFDM in an AWGN and Rayleigh fading channel respectively. Furthermore, this power reallocation policy achieved a throughput of 3.9 (bps/subcarrier) at SNR values as low as $6.5 \mathrm{~dB}$ and $12 \mathrm{~dB}$ in AWGN and Rayleigh fading channels respectively. As is expected from OFDM-SPM, a complete doubling of the system throughput is achieved at higher values of SNR for this case as well.

Comparing the proposed scheme with conventional OFDM with 16-QAM, it can be seen that using the power reallocation policy of OFDM-SPM with QPSK results in a near identical performance to that of conventional OFDM with 16-QAM, while using only half the power. In a Rayleigh fading channel, OFDM-SPM provides a slight gain in terms of BER and throughput over conventional OFDM, however, the opposite is true in an AWGN channel. Overall, this small difference is negligible whereas the power saved is large, giving OFDM-SPM a clear advantage over conventional OFDM.

It is also worth mentioning that the proposed OFDM-SPM with QPSK shows superior performance over MM-OFDMIM and MM-OFDM-IM-IQ [13]. A comparison was done between both of these schemes and OFDM-SPM. In order to ensure a fair comparison, it was made sure that both schemes provide similar throughputs and average energy usage. To this end, the BER of OFDM-SPM with QPSK under power reallocation was compared to that of MMOFDM-IM $(4,8)$ QAM that achieves a maximum throughput of $3.875 \mathrm{bps} / \mathrm{Hz}$, which is nearly equivalent to the throughput achievable by OFDM-SPM with QPSK (4bps/Hz), thus the comparison is in favor of MM-OFDM-IM. In spite of that, it was found that OFDM-SPM achieves a BER of $10^{-2}, 10^{-3}$ and $10^{-4}$ at $16.4 \mathrm{~dB}, 26.5 \mathrm{~dB}$ and $36.5 \mathrm{~dB}$ respectively; whereas MM-OFDM-IM achieves these same BER values at approximately $22.5 \mathrm{~dB}, 28 \mathrm{~dB}$ and $37 \mathrm{~dB}$, respectively, as can be found in [13]. This clearly shows that OFDM-SPM offers a better BER performance than MM-OFDM-IM while also achieving a slightly higher throughout performance. Additionally, the BER and throughput results of OFDMSPM with QPSK under the power reallocation scheme were compared with MM-OFDM-IM-IQ with $(2,4)$ PAM [13] which also achieves a maximum throughput of $4 \mathrm{bps} / \mathrm{Hz}$. The results show that OFDM-SPM with QPSK displays a better BER performance up until a certain point, which is approximately the $35 \mathrm{~dB}$ mark, after which MM-OFDM-IMIQ displays a slightly better BER performance. To illustrate the performance, we present the following remarks. OFDMSPM achieves a BER of $10^{-2}, 10^{-3}$ and $10^{-4}$ at $16.4 \mathrm{~dB}, 26.5$ $\mathrm{dB}$ and $36.5 \mathrm{~dB}$ respectively. On the other hand, MM-OFDMIM-IQ achieves these same BER values at approximately 21 $\mathrm{dB}, 27.5 \mathrm{~dB}$ and $35 \mathrm{~dB}$. The throughput results furthermore, show that OFDM-SPM has a major clear and consistent advantage over MM-OFDM-IM-IQ, Where MM-OFDM-IMIQ achieves throughput values of 3, 3.5 and 4 bits $/ \mathrm{s} / \mathrm{Hz}$ at SNR values of 12,15 and $27 \mathrm{~dB}$, whereas OFDM-SPM achieves a throughput of $3.26 \mathrm{bits} / \mathrm{s} / \mathrm{Hz}$ at $0 \mathrm{~dB}$, and achieves values of 3.5 and $4 \mathrm{bits} / \mathrm{s} / \mathrm{Hz}$ at SNR values of 3.2 and $12 \mathrm{~dB}$ respectively. These results, in addition to the fact that OFDMSPM offers a highly less complex transceiver structure highlights the superiority of OFDM-SPM over MM-OFDM-IM and MM-OFDM-IM-IQ systems. 


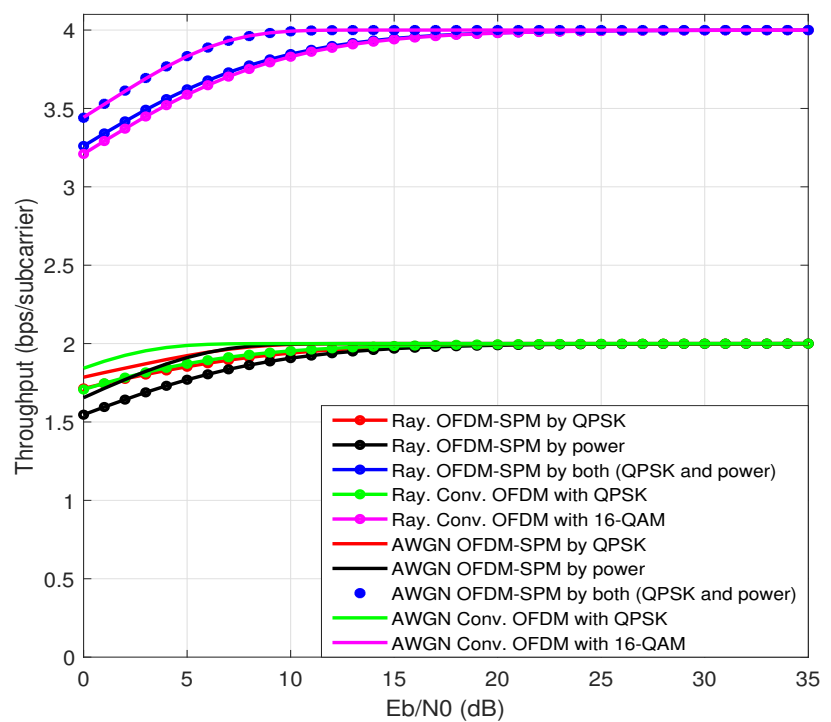

FIGURE 10. Throughput of OFDM-SPM with optimized power reallocation

\section{FAIR COMPARISON BETWEEN OFDM-SPM WITH QPSK AND CONVENTIONAL OFDM WITH 16-QAM}

The comparison between OFDM-SPM and conventional OFDM with 16-QAM is a justified one since both schemes achieve a similar bit transmission rate per symbol. In order to make this comparison a fair one, the average power per OFDM-SPM with QPSK block was made equal to that of a conventional OFDM with 16-QAM block. This was done by setting the $H$ and $L$ power levels according to Eq. (3), where these values were optimized furthermore and found as 2.7 and 0.8426 respectively. The results are displayed and discussed below.

Fig. 11 clearly shows that OFDM-SPM with QPSK is superior to conventional OFDM with 16-QAM both in BER and throughput, where OFDM-SPM achieves a 2.5-3dB gain over conventional OFDM in both an AWGN and Rayleigh fading channel while using the same transmit power.

In terms of throughput, Fig. 12 shows that OFDM-SPMQPSK overall provides a better throughput than conventional OFDM with 16-QAM, especially at low SNR values. As OFDM-SPM achieves a throughput of 3.9 (bps/subcarrier) at an SNR of $3.2 \mathrm{~dB}$ and a throughput 4 (bps/subcarrier)at $9 \mathrm{~dB}$, compared to that of $6.5 \mathrm{~dB}$ and $13 \mathrm{~dB}$ of conventional OFDM with 16-QAM, thus indicating that OFDMSPM approximately brings a $3-4 \mathrm{~dB}$ gain in throughput. Additionally, at $0 \mathrm{~dB}$, OFDM-SPM achieves throughputs of 3.69 (bps/subcarrier) and 3.49 (bps/subcarrier) compared to 3.44 (bps/subcarrier) and 3.21 (bps/subcarrier) of conventional OFDM with 16-QAM, in AWGN and Rayleigh fading channels, respectively.

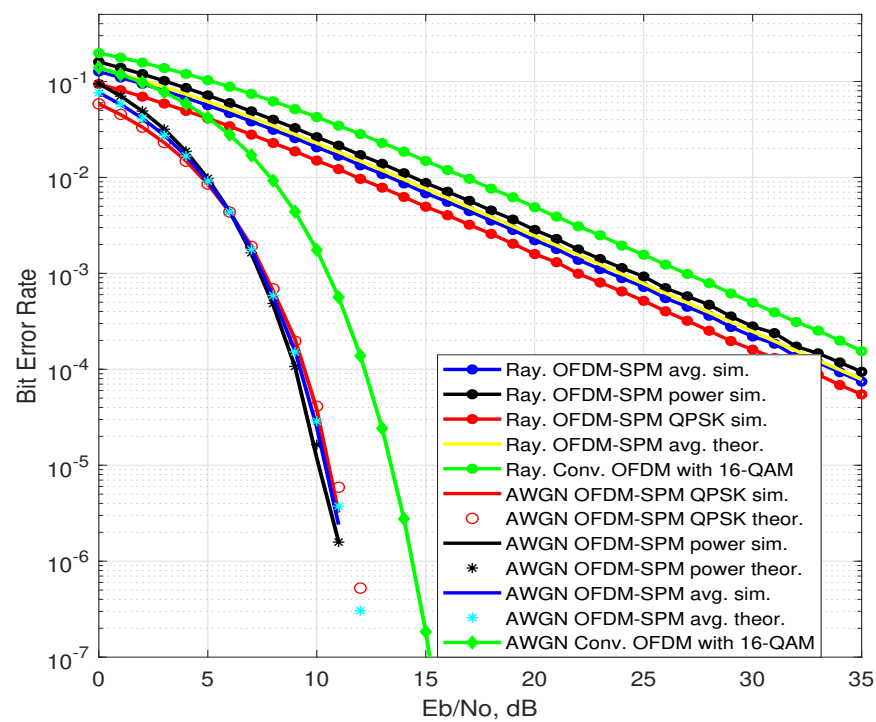

FIGURE 11. BER comparison between OFDM-SPM and OFDM with 16-QAM

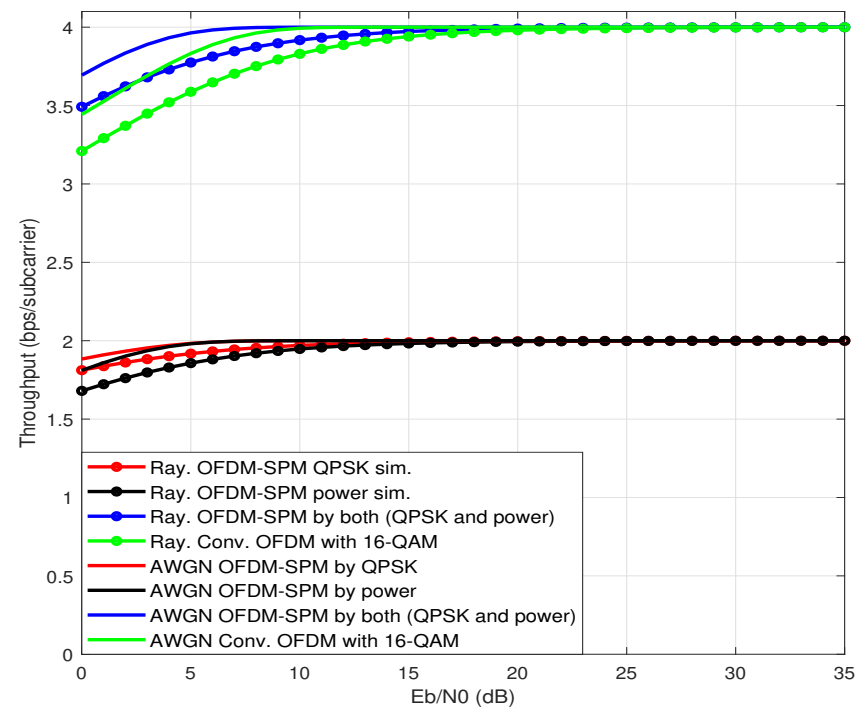

FIGURE 12. BER comparison between OFDM-SPM and OFDM with 16-QAM

Given four different cases that were displayed, OFDMSPM managed to prove its ability in doubling the spectral efficiency, and thus data rate of a communication system even when paired with a two dimensional higher order symbol modulation scheme. Following the simulation results, it can be said that the spectral gain that OFDM-SPM offers is independent of the modulation scheme order, unlike OFDM-IM, OFDM-SNM, and other modulation schemes in which the spectral gain offered by the third dimension they introduce deteriorates as the modulation order becomes higher, this is because OFDM-SPM utilizes all the subcarriers of the OFDM symbol, and does not base its spectral gain on trading 
the gain of a subcarrier for the gain of another dimension. Rather, OFDM-SPM completely adds the gain of the third dimension it introduces to the gain of the subcarriers of the OFDM block fully.

\section{CONCLUSION}

In this paper, OFDM-SPM was paired with a two dimensional symbol modulation technique, namely QPSK in which both the in-phase and quadrature subcarriers are utilized to carry data. By manipulating the power of the subcarriers of the OFDM block, OFDM-SPM with BPSK is able to attain a doubling in the spectral efficiency and data rate of the system. In order to investigate the consistency and integrity of the gains of OFDM-SPM when paired with higher order two dimensional symbol modulation schemes, OFDM-SPM was paired with QPSK symbol modulation. Since QPSK uses both in-phase and quadrature subcarriers, OFDM-SPM is able to manipulate the power of both components of the subcarriers of the OFDM block, namely the in-phase and quadrature components.

It was found ultimately, that the gain of OFDM-SPM persists even when paired with higher order modulation schemes, thus characterizing OFDM-SPM uniquely as a scheme which adds a third dimension with a spectral gain independent of the modulation order. This was made evident by the results displayed in the performance demonstration section. In particular, OFDM-SPM was able to achieve a doubling of the system throughput at high values of SNR regardless of the power policy followed and regardless of the channel model.

Furthermore, OFDM-SPM achieved a much higher throughput than that of conventional OFDM even at SNR values as low as $5 \mathrm{~dB}$ in both AWGN and Rayleigh fading channels. Although OFDM-SPM exhibits a BER degradation When compared to conventional OFDM, the merits it introduces offer a worthy compensation. When comparing OFDM-SPM with QPSK to conventional OFDM with QPSK, OFDM-SPM offers reduction of half the transmit power, doubling the throughput, more flexibility in providing different power reallocation policies, less transmission times due to the use of fewer subcarriers which translate to fewer resources in the frequency and time domain, and the possibility of reducing the system complexity if half the IFFT size is to be used. The degradation in the BER can be treated by using the reallocation policy initially; furthermore, it is notable to mention that other methods in conjunction with OFDM-SPM can be used to improve the BER performance such as using coding techniques and antenna receiver diversity schemes; however, this is left for future research works and studies.

As OFDM-SPM achieves a data rate similar to that of conventional OFDM with 16-QAM, it was found that OFDMSPM is able to achieve a performance similar to that of conventional OFDM with 16-QAM while saving half the power. Furthermore, a fair comparison was done between these two schemes by equalizing the average power per OFDM block of both schemes. It was found that OFDM-SPM-QPSK dis- plays superiority to conventional OFDM with 16-QAM in both BER and throughput, where OFDM-SPM displayed a significant gain (2.5-3 dB) over its counterpart.

Other merits of OFDM-SPM as has been previously mentioned in the literature is that OFDM-SPM avoids many of the flaws which are characteristic to other previously proposed techniques, such as the error propagation in OFDM-SIM and the complexity of the receiver in OFDM-IM. The complexity that OFDM-SPM with QPSK adds to the system transceiver structure is minor and in fact can reduce the complexity further by reducing the IFFT size for achieving the same throughput that conventional OFDM can deliver.

Additionally, OFDM-SPM can be seen as a scheme capable of transmitting two separate data streams. By observing the BER curves of OFDM-SPM regardless of the power scheme followed, it is seen that one stream of bits is more erroneous than the other. This introduces the possibility of using OFDM-SPM to serve different users with different bit error rate requirements. These properties of OFDMSPM and the findings of OFDM-SPM with QPSK signify that more importance and research should be done on this scheme, as the gains are promising. Furthermore, the results are indicative of OFDM-SPM being a candidate fit for the requirements of $6 \mathrm{G}$ and beyond communication systems. This is so because OFDM-SPM as per the previous analysis was able to bring obvious advantages in terms of latency, throughput, reliability, complexity and power saving, which are all critical metrics that will ultimately determine future $6 \mathrm{G}$ technologies.

\section{FUTURE RESEARCH DIRECTION}

Given the observed gains of OFDM-SPM, there is much future work to be done to improve this scheme and further affirm its capability of being a modulation scheme to be utilized in $6 \mathrm{G}$ and beyond communication systems. One of the topics that future research should specifically focus on is investigating the performance of OFDM-SPM with higher order modulation schemes such as higher order M-PSK. This is a very important future research direction because employing OFDM-SPM to double the spectral efficiency of 8-PSK, 16-PSK and higher modulation schemes would require the formulation of a greater number of subcarrier power levels, namely $\log _{2} M$ power levels. Particularly, when employing M-PSK symbol modulation, this would furthermore require the allocation of $n \log _{2} M$ to be assigned as the number of bits to determine the power levels of the subcarriers. As such, the integrity and performance of the scheme, would then highly rely upon determining the optimal power levels for each modulation order, which is why a separate dedicated research on this topic will be of critical importance in determining the validity of the adoption of OFDM-SPM in future communication systems.

Additionally, other future topics which should be studied are additional techniques which could be paired with OFDMSPM to improve its performance, both in terms of bit error rate and spectral efficiency. Whether techniques which have 
been applied to other schemes can be applied equivalently to OFDM-SPM, replicating similar results remains an issue to be investigated. For example, techniques which utilize pre-coding have been paired with both SM-OFDM [29] and OFDM-IM [30] systems respectively to improve BER performance. Pairing techniques such as these with OFDM-SPM, if integrated properly can enhance its performance. This improvement in BER performance could allow OFDM-SPM to achieve a doubling in the spectral efficiency at a minor cost in terms of BER degradation, thus making OFDM-SPM applicable to a vast number of application scenarios and truly emphasizing its candidacy as a technique to be used in $6 \mathrm{G}$ and beyond communication systems.

It has been recently reported in the literature furthermore, that both the concepts of SM-OFDM and NOMA have been paired to introduce a novel cooperating relay system which uses SM-aided NOMA [31]. This novel system was able to achieve a superior BER performance when compared to other preceding schemes within the same structural context. It can be said however, that while both this system and OFDM-SPM use power as a main characteristic which can be manipulated to improve the spectral efficiency of their respective schemes, the two schemes when compared are both structurally and conditionally different, thus disallowing a direct comparison between these schemes. On the other hand, the work in [31] brings forth a possible subject of future research, which has yet to be investigated, that is whether OFDM-SPM can be integrated efficiently with NOMA to yield a more productive and promising scheme.

Being a novel scheme, it is of utmost importance to truly understand the magnitude of the advantages of using OFDMSPM and of employing it in future generation communication systems.

\section{APPENDIX: OFDM-SPM THEORETICAL BER ANALYSIS}

\section{A. DETECTION OF THE QPSK SYMBOLS OF OFDM-SPM}

As OFDM-SPM dictates, the power of the in-phase and quadrature subcarriers is varied according to the incoming stream of data bits. The effect of the variation of this power extends to the QPSK symbols as they are carried by their corresponding subcarriers. As such, the BER expression of the OFDM-SPM QPSK symbols is different from that of conventional OFDM with QPSK.

By varying the power of the subcarriers, the Euclidian distance between the two symbols also changes as is made evident by Fig. 2. As is known, the BER of QPSK is statistically determined by the minimum Euclidian distance between two adjacent symbols. Furthermore, since QPSK consists of two orthogonal BPSK schemes, one can treat the BER expressions of QPSK and BPSK to be equivalent. As such, in order to derive the BER expression of QPSK when used with OFDM-SPM, we start from the BER expression of BPSK in a Rayleigh fading channel, which is our channel of interest, which is given by Eq. (24)

$$
B E R=\frac{1}{2}\left(1-\sqrt{\frac{\frac{E_{b}}{N_{0}}}{1+\frac{E_{b}}{N_{0}}}}\right)
$$

Eq. (24) shows the BER expression of a BPSK symbol with energy $\frac{E_{b}}{N_{0}}$. In OFDM-SPM, the energy of the symbol is changed to either high or low according to the power of the subcarrier which carries it. As such, a multiplication factor must be introduced to Eq. (24) to compensate for variation in the energy of the symbol. As $H$ and $L$ are factors which determine the amplitude of a symbol with regards to the given amplitude of a BPSK symbol, these factors can be included in Eq. (24), to simulate the necessary change. However, these factors will be squared in order to represent energy rather than amplitude, resulting in the following equations for the BER of OFDM-SPM with QPSK, for the high power and low power symbols respectively.

$$
\begin{aligned}
& B E R_{H}=\frac{1}{2}\left(1-\sqrt{\frac{H^{2} \frac{E_{b}}{N_{0}}}{1+H^{2} \frac{E_{b}}{N_{0}}}}\right) \\
& B E R_{L}=\frac{1}{2}\left(1-\sqrt{\frac{L^{2} \frac{E_{b}}{N_{0}}}{1+L^{2} \frac{E_{b}}{N_{0}}}}\right)
\end{aligned}
$$

Additionally, since the probability of occurrence of a high power or low power symbol is equal, and given that we must take both the errors due to high power and low power QPSK symbols into account, the total BER expression for the detection of the QPSK symbols in OFDM-SPM is given as the statistical average of Eq. (25) and Eq. (26) as follows:

$$
B E R_{Q P S K}=\frac{B E R_{L}+B E R_{H}}{2}
$$

Leading us to the same expressions found in Eq. 16 - 18.

\section{B. DETECTION OF THE OFDM-SPM POWER BITS}

By referring to Fig. 2, we can use the constellation points of OFDM-SPM to derive the analytical expression of the bit error rate of OFDM-SPM. By looking at Fig. 2, it is intuitive to assume the derivation of the BER expression of OFDMSPM will be done in a manner similar to that of 4-PAM. Similar to the case of 4-PAM, the factors affecting the power of an OFDM-SPM symbol is the AWGN and the channel response. Thus, the changes exhibited in the BER expressions of OFDM-SPM will be to simulate the effect of the different Euclidean distances between the symbols.

Since the constellation points on the right and left halves of Fig. 2 are symmetric, we can simply find the probability of error of the points on either half of the constellation diagram and then multiply the collective result by a factor of 2. Arbitrarily, for demonstration purposes, we choose the right half of the constellation map. 
Our starting point is by observing, that the probability of a BPSK symbol with energy $\frac{E_{b}}{N_{0}}$ being found in error due to the effects of noise and the channel response is given by the following equation [26]:

$$
B E R=\frac{1}{2}\left(1-\sqrt{\frac{\frac{E_{b}}{N_{0}}}{1+\frac{E_{b}}{N_{0}}}}\right),
$$

where the Euclidean distance between the symbols is a function of ratio between the energy of the symbols and noise variance $\frac{E_{b}}{N_{0}}$. This equation can be used as a basis for finding the bit error rates of the power detection process of the symbols of OFDM-SPM.

Generally, we have two cases of error in the right half of the constellation map, namely Fig. 2. Particularly, either a low power symbol is wrongly detected as a high power symbol, or that a high power symbol is detected as a low power symbol.

Firstly, we consider the case of a low power symbol being detected as a high power symbol. This can occur in one of two ways, either the symbol is detected as a high power symbol with amplitude $\left(H \sqrt{E_{b}}\right)$, with a probability of occurrence which can be found similar to that of Eq. (15), with a small change to simulate the change in the Euclidean distance between the two symbols. The minimum distance for the symbol to be detected as a symbol of amplitude $\left(H \sqrt{E_{b}}\right)$ is $\frac{H-L}{2}$. As such, the probability of this occurring, let's call it $E_{l}$ is given as:

$$
E_{1}=\frac{1}{2}\left(1-\sqrt{\frac{\left(\frac{H-L}{2}\right)^{2} \frac{E_{b}}{N_{0}}}{1+\left(\frac{H-L}{2}\right)^{2} \frac{E_{b}}{N_{0}}}}\right)
$$

It is also possible that the low power symbol can be detected as a high power symbol with amplitude $\left(-H \sqrt{E_{b}}\right)$ which is less likely to occur but still possible. The minimum distance for the low power symbol to be detected as such is $\frac{H+3 L}{2}$, which gives us a probability of occurrence $E_{2}$ :

$$
E_{2}=\frac{1}{2}\left(1-\sqrt{\frac{\left(\frac{H+3 L}{2}\right)^{2} \frac{E_{b}}{N_{0}}}{1+\left(\frac{H+3 L}{2}\right)^{2} \frac{E_{b}}{N_{0}}}}\right)
$$

Assuming an equal probability of occurrence of 0 's and 1 's, the probability of the occurrence of such a symbol is $\frac{1}{4}$, giving the total probability of error as:

$$
\frac{1}{4}\left(E_{1}+E_{2}\right)
$$

Now, we come to the probability of a high power symbol being detected as a low power symbol. Starting from the symbol with amplitude $\left(H \sqrt{E_{b}}\right)$, we see that the power bit conveying the power of this symbol will be detected in error if the symbol is detected as a low power symbol in either half of the constellation plane; however, if the noise is high enough such that the symbol is detected as a symbol with amplitude $\left(-H \sqrt{E_{b}}\right)$, the power bit detected will not be in error as the power of the symbol remains high. We can take account of this by subtracting the probability of this occurring from the probability of the symbol being detected as a low power symbol. The minimum distance required for the high power symbol to be detected as a low power symbol is given as $\frac{H-L}{2}$, thus we obtain the probability of this occurrence $E_{3}$ as:

$$
E_{3}=\frac{1}{2}\left(1-\sqrt{\frac{\left(\frac{H-L}{2}\right)^{2} \frac{E_{b}}{N_{0}}}{1+\left(\frac{H-L}{2}\right)^{2} \frac{E_{b}}{N_{0}}}}\right),
$$

which is identical to Eq. (16). The minimum distance required for the high power symbol in the right half of the constellation plane to be detected as a high power symbol in the left half plane is $\frac{3 H+L}{2}$. As such, the probability of this occurrence $E_{4}$ is found as

$$
E_{4}=\frac{1}{2}\left(1-\sqrt{\frac{\left(\frac{3 H+L}{2}\right)^{2} \frac{E_{b}}{N_{0}}}{1+\left(\frac{3 H+L}{2}\right)^{2} \frac{E_{b}}{N_{0}}}}\right)
$$

Similar to the previous case, the probabilities are multiplied by a factor of $\frac{1}{4}$, and we obtain the following:

$$
\frac{1}{4}\left(E_{3}-E_{4}\right)
$$

The total probability of error is thus a sum of the terms in Eq. (18) and Eq. (21). Additionally, since we assumed symmetry between the symbols of the left half and right of the constellation plane, and because the terms $E_{1}$ and $E_{3}$ are identical, they can be summed. Thus, we are then left with

$$
E_{1}+\frac{1}{2} E_{2}-\frac{1}{2} E_{4}
$$

which is identical to the expression given by Eq. (10).

The same manner of derivation can be followed for the case of the OFDM-SPM BER due to the bits carried by the power of the subcarriers in an AWGN channel by starting from the BER equation of BPSK below, instead of Eq. (28).

$$
B E R_{B P S K}=\frac{1}{2} \operatorname{erfc}\left(\sqrt{\frac{E_{b}}{N_{0}}}\right)
$$

\section{REFERENCES}

[1] Y. Belallou, J. M. Hamamreh, and A. Hajar, "OFDM-subcarrier power modulation with two dimensional signal constellations," in 2019 Innovations in Intelligent Systems and Applications Conference (ASYU), Oct 2019, pp. 1-6.

[2] W. Saad, M. Bennis, and M. Chen, "A vision of $6 \mathrm{G}$ wireless systems: Applications, trends, technologies, and open research problems," pp. 1-9, Feb 2019.

[3] B. Zong, C. Fan, X. Wang, X. Duan, B. Wang, and J. Wang, "6G technologies: Key drivers, core requirements, system architectures, and enabling technologies," IEEE Vehicular Technology Magazine, vol. 14, no. 3, pp. 18-27, 2019.

[4] Y. Liu, Z. Qin, M. Elkashlan, Y. Gao, and A. Nallanathan, "Nonorthogonal multiple access in massive MIMO aided heterogeneous networks," in 2016 IEEE Global Communications Conference (GLOBECOM), Dec 2016, pp. 1-6.

[5] A. Sabharwal, P. Schniter, D. Guo, D. W. Bliss, S. Rangarajan, and R. Wichman, "In-band full-duplex wireless: Challenges and opportunities," IEEE Journal on Selected Areas in Communications, vol. 32, no. 9 , pp. 1637-1652, Sep. 2014. 
[6] A. Jaradat, J. Hamamreh, and H. Arslan, "Modulation options for OFDMbased waveforms: Classification, comparison, and future directions," IEEE Access, vol. 7, pp. 17 263-17 278, Jan 2019.

[7] R. Y. Mesleh, H. Haas, S. Sinanovic, C. W. Ahn, and S. Yun, "Spatial modulation," IEEE Transactions on Vehicular Technology, vol. 57, no. 4, pp. 2228-2241, 2008.

[8] R. Abu-alhiga and H. Haas, "Subcarrier-index modulation OFDM," in 2009 IEEE 20th International Symposium on Personal, Indoor and Mobile Radio Communications, Sep. 2009, pp. 177-181.

[9] E. Basar, Ü. Aygölü, E. Panayirci, and H. V. Poor, "Orthogonal frequency division multiplexing with index modulation,” 2012 IEEE Global Communications Conference (GLOBECOM), pp. 4741-4746, 2012.

[10] A. M. Jaradat, J. M. Hamamreh, and H. Arslan, "OFDM with subcarrier number modulation," IEEE Wireless Communications Letters, vol. 7, no. 6, pp. 914-917, Dec 2018.

[11] N. Ishikawa, S. Sugiura, and L. Hanzo, "Subcarrier-index modulation aided OFDM - will it work?" IEEE Access, vol. 4, pp. 2580-2593, 2016.

[12] E. Basar, M. Wen, R. Mesleh, M. Di Renzo, Y. Xiao, and H. Haas, "Index modulation techniques for next-generation wireless networks," IEEE Access, vol. 5, pp. 16 693-16 746, 2017.

[13] M. Wen, E. Basar, Q. Li, B. Zheng, and M. Zhang, "Multiple-mode orthogonal frequency division multiplexing with index modulation," IEEE Transactions on Communications, vol. 65, no. 9, pp. 3892-3906, Sep. 2017.

[14] T. Mao, Z. Wang, Q. Wang, S. Chen, and L. Hanzo, "Dual-mode index modulation aided OFDM," IEEE Access, vol. 5, pp. 50-60, 2017.

[15] X. Cheng, M. Zhang, M. Wen, and L. Yang, "Index modulation for 5G: Striving to do more with less," IEEE Wireless Communications, vol. 25, no. 2, pp. 126-132, April 2018.

[16] T. Datta, H. S. Eshwaraiah, and A. Chockalingam, "Generalized spaceand-frequency index modulation," IEEE Transactions on Vehicular Technology, vol. 65, no. 7, pp. 4911-4924, July 2016.

[17] M. Wen, X. Cheng, and L. Yang, "Index modulation for 5G wireless communications," Dec 2017.

[18] M. Wen, B. Zheng, K. J. Kim, M. Di Renzo, T. A. Tsiftsis, K. Chen, and N. Al-Dhahir, "A survey on spatial modulation in emerging wireless systems: Research progresses and applications," IEEE Journal on Selected Areas in Communications, vol. 37, no. 9, pp. 1949-1972, 2019.

[19] J. Zhang, Y. Wang, J. Zhang, and L. Ding, "Polarization shift keying (polarsk): System scheme and performance analysis," IEEE Transactions on Vehicular Technology, vol. 66, no. 11, p. 10139-10155, Nov 2017.

[20] T. Mao, Q. Wang, Z. Wang, and S. Chen, "Novel index modulation techniques: A survey," IEEE Communications Surveys Tutorials, vol. 21, no. 1, pp. 315-348, 2019.

[21] A. Khandani, "Media-based modulation: A new approach to wireless transmission," Jul 2013, pp. 3050-3054.

[22] S. Dang, G. Ma, B. Shihada, and M. Alouini, "Enhanced orthogonal frequency-division multiplexing with subcarrier number modulation," IEEE Internet of Things Journal, pp. 1-1, 2019.

[23] F. Halabi, L. Chen, S. Parre, S. Barthomeuf, R. P. Giddings, C. AupetitBerthelemot, A. Hamié, and J. M. Tang, "Subcarrier index-power modulated optical OFDM and its performance in IMDD PON systems," Journal of Lightwave Technology, vol. 34, no. 9, pp. 2228-2234, May 2016.

[24] A. Hajar, J. M. Hamamreh, M. Abewa, and Y. Belallou, "A spectrally efficient OFDM-based modulation scheme for future wireless systems," in 2019 Scientific Meeting on Electrical-Electronics Biomedical Engineering and Computer Science (EBBT), April 2019, pp. 1-4.

[25] J. M. Hamamreh, A. Hajar, and M. Abewa, "Orthogonal frequency division multiplexing with subcarrier power modulation for doubling the spectral efficiency of $6 \mathrm{G}$ and beyond networks," Transactions on Emerging Telecommunications Technologies, vol. 31, no. 4, p. e3921, 2020.

[26] J. M. Hamamreh, E. Basar, and H. Arslan, "OFDM-subcarrier index selection for enhancing security and reliability of 5G URLLC services," IEEE Access, vol. 5, pp. 25 863-25 875, 2017.

[27] Z. Hu, F. Chen, M. Wen, F. Ji, and H. Yu, "Low-complexity LLR calculation for ofdm with index modulation," IEEE Wireless Communications Letters, vol. 7, no. 4, pp. 618-621, 2018.

[28] D. Tsonev, S. Sinanovic, and H. Haas, "Enhanced subcarrier index modulation (SIM) OFDM," in 2011 IEEE GLOBECOM Workshops (GC Wkshps), Dec 2011, pp. 728-732.

[29] J. Li, M. Wen, X. Cheng, Y. Yan, S. Song, and M. H. Lee, "Generalized precoding-aided quadrature spatial modulation," IEEE Transactions on Vehicular Technology, vol. 66, no. 2, pp. 1881-1886, 2017.
[30] S. Dang, G. Ma, B. Shihada, and M. Alouini, "Enhanced orthogonal frequency-division multiplexing with subcarrier number modulation," IEEE Internet of Things Journal, vol. 6, no. 5, pp. 7907-7920, 2019.

[31] Q. Li, M. Wen, E. Basar, H. V. Poor, and F. Chen, "Spatial modulationaided cooperative NOMA: Performance analysis and comparative study,' IEEE Journal of Selected Topics in Signal Processing, vol. 13, no. 3, pp. 715-728, 022019.

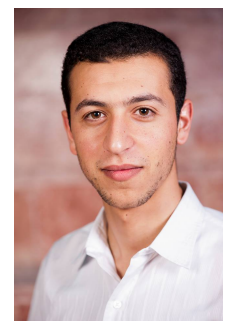

JEHAD M.HAMAMREH received the B.Sc. degree in electrical and telecommunication engineering from An-Najah University, Nablus, in 2013, and the Ph.D. degree in electrical electronics engineering and cyber systems from Istanbul Medipol University, Turkey, in 2018. He was a Researcher with the Department of Electrical and Computer Engineering, Texas A\&M University. $\mathrm{He}$ is currently an Assistant Professor with the Electrical and Electronics Engineering Department, Antalya International (Bilim) University, Turkey. His current research interests include wireless physical and MAC layers security, orthogonal frequency-division multiplexing multiple-input multiple-output systems, advanced waveforms design, multi-dimensional modulation techniques, and orthogonal/non-orthogonal multiple access schemes for future wireless systems. He is a Regular Reviewer for various peer-reviewed journals as well as a TPC Member for several international conferences. He is very open for collaboration and can be reached via email at jehad.hamamreh@gmail.com.

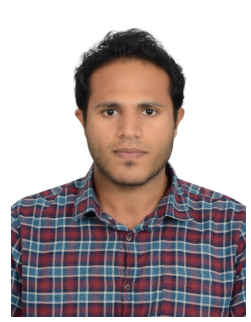

ABDULWAHAB HAJAR received his B.Sc from the department of electrical and electronics engineering, Antalya Bilim University in Antalya, Turkey, in 2019.

$\mathrm{He}$ is an author of several published research papers, and is working with professor Jehad M. Hamamreh on the topic of OFDM-SPM for Future $6 \mathrm{G}$ and Beyond Networks. 